\title{
Evolución de las prácticas profesionales contables en el relacionamiento de los stakeholders, 2014-2018
}

\author{
Evolution of professional practices accounts in \\ your relationship stakeholders, 2014-2018
}

Recibido: 12-11-2019 • Aprobado: 29-10-2020 • Página inicial: 197 - Página final: 223

\author{
Juan Camilo Franco Jaramillo* \\ José Hermes Hidalgo Giraldo** \\ Carlos Eduardo Castaño Ríos***
}

\begin{abstract}
Resumen: este artículo tiene como objetivo evaluar la evolución de las prácticas profesionales contables en relación con los stakeholders en un período comprendido entre 2014 al 2018. La metodología aplicada a este estudio es cualitativa, basada en la revisión documental y entrevistas a nueve contadores en ejercicio, con más de cuatro años de experiencia a la fecha. Arrojando resultados que demuestran que las prácticas contables presentaron cambios importantes en el período estudiado con relación a trabajos anteriores y que, además, se tiene una perspectiva de cambio permanente frente a los diferentes requerimientos de información con ética y responsabilidad social necesarios en la transición de las empresas a la implementación del nuevo marco técnico normativo contable.
\end{abstract}

Palabras clave: prácticas profesionales contables; nuevo marco normativo contable; stakeholders; Contaduría Pública.

\begin{abstract}
This article aims to evaluate the evolution of professional accounting practices in relation to stakeholders in a period from 2014 to 2018. The methodology applied to this study is qualitative, based on the documentary review and interviews with nine practicing accountants, with more than four years of experience to date. Throwing results that show that accounting practices presented important changes in the period studied in relation to previous works and that, in addition, there is a perspective of permanent change in the face of the different information requirements with ethics and social responsibility necessary in the transition of the companies to the implementation of the new technical accounting regulatory framework.
\end{abstract}

Keywords: Professional accounting practices; new accounting regulatory framework; stakeholders; public accountancy.

\footnotetext{
* Contador Público de la Universidad de Antioquia y Especialista en Legislación Tributaria de la Universidad Autónoma Latinoamericana. Estudiante de la Maestría en Contabilidad Financiera y de Gestión de la Universidad de Antioquia, Colombia. juancamilofj@hotmail.com ORCID: https://orcid.org/0000-0002-8494-8717

** Contador Público de la Universidad Cooperativa de Colombia y Especialista en Revisoría Fiscal de la Universidad Autónoma Latinoamericana. Estudiante de la Maestría en Contabilidad Financiera y de Gestión de la Universidad de Antioquia, Colombia. josehermeshidalgogiraldo@gmail.com ORCID: https://orcid.org/0000-0002-4589-8016

*** Contador Público y Magíster en Administración de la Universidad de Antioquia. Jefe del Centro de Investigaciones y Consultorías - CIC, de la Facultad de Ciencias Económicas de la Universidad de Antioquia. Integrante del Grupo de Investigaciones y Consultorías en Ciencias Contables - GICCO de la mima universidad. eduardo.castano@udea.edu.co ORCID: https://orcid.org/0000-0003-4117-0375
} 


\section{Evolução das práticas profissionais contas em seu relacionamento partes interessadas, 2014-2018}

Resumo: este artigo tem como objetivo avaliar a evolução das práticas contábeis profissionais em relação ás partes interessadas no período de 2014 até 2018. A metodologia aplicada neste estudo é qualitativa, com base na revisão documental e entrevistas com nove contadores em atividade, com mais de quatro anos de experiência até o momento. Produzindo resultados que mostram que as práticas contábeis apresentam mudanças importantes no período estudado em relação aos trabalhos anteriores e que, além disso, há uma perspectiva de mudança permanente frente aos diversos requisitos de informação com ética e responsabilidade social necessários na transição das empresas para a implementação do novo quadro regulamentar técnico de contabilidade.

Palavras-chave: práticas contábeis profissionais; novo marco regulatório contábil; partes interessadas; contabilidade pública. 


\section{Introducción}

El desarrollo de las prácticas contables ha estado ligado a la evolución de la humanidad, bajo la premisa de la necesidad de información para la toma de decisiones. El nacimiento mismo de la contabilidad ha sido producto del requerimiento social de dar cuenta de bienes, hechos y recursos que rodean la actividad económica y social. Según Gertz Manero (1976), para hablar de contabilidad, se requerían al menos tres momentos clave en la historia: primero, el asentamiento del hombre en un territorio específico; segundo, la aparición de la escritura y la unidad de medida como elementos para asegurar el control de recursos; tercero, un volumen importante de actividades económicas que superaban la capacidad de procesamiento de información de la memoria humana.

Para Hernández (2002), "la contabilidad es una actividad tan antigua como la humanidad misma" (p.1) y se ha reconocido que desde que el hombre sabe de su existencia requería llevar cuentas sobre lo que cazaba, consumía, almacenaba, entre otras actividades. En esa vía, Gertz Manero (1976) plantea que el desarrollo histórico de la contabilidad parte del año 6.000 A.C., en donde ya existían elementos necesarios para llevar a cabo la actividad contable como la escritura, los números, el concepto de propiedad, la moneda, el crédito de los mercados, la tablilla de barro. No obstante, nuevos hallazgos arqueológicos posicionan el nacimiento de la contabilidad en África 20.000 años A.C. con la evidencia del hueso de Ishango (Sy, 2014), lo cual reconfigura lo que hasta el momento se conocía sobre la historia de la contabilidad ampliando el espectro a un período de tiempo mucho más largo en el desarrollo de la humanidad.

Momentos más recientes de la historia de la contabilidad permiten comprender el tránsito entre la contabilidad memorial, la simple y la partida doble, desde el punto de vista del reconocimiento y registro de hechos económicos, cambios que estuvieron marcados por la evolución y mayor complejidad del comercio, la creación de las figuras societarias e incremento de las operaciones de financiación. En este contexto, se ha podido evidenciar que uno de los objetivos de la contabilidad es brindar información para la toma de decisiones y el control de los recursos, aunque con un mayor interés por los dueños del capital y del Estado (como fuente de control de la economía).

Así, las prácticas contables han evolucionado a la par que la disciplina; sin embargo, el marcado interés para mostrar resultados exclusivamente a los inversionistas y al Estado, ha llevado a que la formación contable se realice bajo el cumplimiento de marcos regulativos que terminan con la generación de 


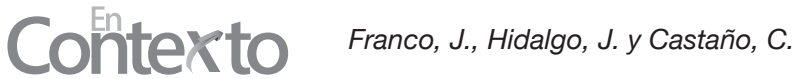

informes, rezagando las funciones del contador, e ingresando la profesión en un descontento y aislamiento frente a los demás grupos de interés que buscan, no solo información financiera, sino aquella que requieren para atender sus necesidades, según al grupo que pertenecen (Freeman, 1984). A razón de lo anterior, surge el cuestionamiento sobre ¿cómo ha sido la evolución de las prácticas profesionales contables en relación con los stakeholders 2014-2018?

Con el desarrollo de este trabajo se pretendió dar continuidad al análisis de la evolución de las prácticas contables en relación con los stakeholders en las empresas privadas colombianas. Este análisis fue tratado inicialmente por Castaño et al. (2015), quienes plantearon ocho perspectivas denominadas cumplimiento legal, TIC, informes contables, análisis de la información para crear valor agregado, teneduría de libros, contador como gerente de la información financiera, ética y perspectiva social de stakeholder, las cuales se retoman para este trabajo y se desarrollan en el período 2014 a 2018, lapsos de amplios cambios liderados principalmente por la llegada de una nueva regulación contable a las organizaciones colombianas.

Igualmente, se desea exhortar con este artículo a todos los vinculados con el ejercicio de la profesión contable, entre ellos: los gremios, el Estado, la academia, los grupos económicos, entidades no gubernamentales, inversionistas y la comunidad para trabajar con el objetivo de mantener unas prácticas contables actualizadas, que atiendan a sus necesidades y las de los diferentes usuarios de la información (stakeholders); lo que muy seguramente aclararía el contenido social de las prácticas contables, enmarcadas en la fe pública del contador.

\section{La profesión contable y sus prácticas}

En el ejercicio de la profesión contable, el contador público debe cumplir con la información y requerimientos de los "terceros interesados" (stakeholders), como lo cita el artículo 35 de la Ley 43 de 1990 (Congreso de la República, 13 de diciembre de 1990), que vincula las actividades empresariales con la su práctica profesional contable.

En sus inicios, las prácticas contables atendían con sus informes los requerimientos de la comunidad, como lo establece el trabajo de Rivera (2013), el cual concluye que la función social que siempre ha tenido la contabilidad es dar cuenta o proporcionar información a las personas. Sin embargo, se ha evidenciado en la historia reciente de la contabilidad un interés primario por inversionistas y el Estado (más como herramienta de control fiscal), situación que debe dar un giro hacia lo social. 
La perspectiva social de la práctica contable dio un giro con la revolución industrial, ligada al desarrollo de los mercados financieros, pues se requerían conocimientos especializados en contabilidad financiera, costos y auditoría de cuentas (Richardson, 2018). Así mismo, la expansión de la profesión se da en el siglo XX, cuando se adopta en el mundo un modelo similar al anglosajón para la financiación de las actividades económicas.

En este sentido, los últimos 200 años han traído consigo una constante evolución de la profesión contable y sus prácticas aunado a la transformación misma de la sociedad y sus formas de relacionamiento. De allí, la importancia de revisar constantemente las prácticas contables y sus redefiniciones en el contexto social.

Para comprender la práctica profesión contable, lo primero que hay que plantear es que la misma se inscribe dentro de las actuaciones dentro de una sociedad. Una práctica, según Reckwitz (2002) es

[...] una forma rutinizada de conducta que está compuesta por distintos elementos interconectados: actividades del cuerpo, actividades mentales, objetos y uso, y otras formas de conocimiento que están en la base tales como significados, saberes prácticos, emociones y motivaciones [...], la práctica forma una unidad cuya existencia depende de la interconexión especifica entre estos distintos elementos (p.249).

En este orden de ideas, la práctica contable se ha entendido de forma genérica como un "[...] proceso de registro y comunicación de transacciones, enfocado hacia la representación de la realidad económica y financiera de un ente" (Ámez, 1999, p.57).

Igualmente, no se puede dejar de analizar que en todo momento es una persona la que realiza la práctica contable y, como tal, está influenciada por unos fundamentos internos y unas exigencias externas, las cuales pueden llegar a condicionar su actuar (MacIntyre, 2004).

La práctica contable asume la multiplicidad de conocimientos que debe poseer un contador público para poder ejercer y realizar su trabajo de tal forma, que pueda atender a todos sus clientes e igualmente con la capacidad de ir evolucionando en conocimiento, en la medida que el ejercicio y el entorno lo exijan, así lo reconoce la misma Ley 43 de 1990 en su artículo 35. 


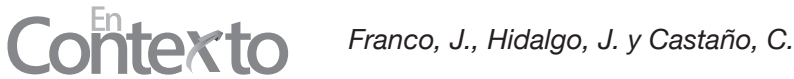

El ejercicio de la práctica contable debe satisfacer a los terceros interesados, término que fue utilizado y definido por Freeman (1984), con lo cual se espera que efectivamente los distintos agentes interesados sean incluidos en los informes empresariales. En la actualidad se tiene una teoría de los stakeholders que ha trascendido con fuerza estos primeros postulados y que es una forma de dar legitimidad a las organizaciones frente a la sociedad (Larrinaga, 2017).

Se requiere, además, que el contador logre el perfil que la sociedad requiere. En este sentido, el profesor Araujo expresa que "[...] el contador público debe ser, ante todo, una persona que siente, alguien integrante de la sociedad, debe ser una persona con capacidad profesional, con una formación integral, con una capacidad de liderazgo, con responsabilidad social y con espíritu investigativo" (1995, pp.164-165).

Vale la pena indicar, que se entiende por práctica profesional contable

[...] al quehacer del contador público, a sus tareas, a sus actividades, a la ejecución de su trabajo en la organización. Dado lo anterior, lo que se entienda por práctica profesional contable dependerá en gran medida de las necesidades que existan en un momento determinado o en una organización en particular, lo que enfocará el trabajo del contador público hacia una menor o mayor cobertura (Castaño et al., 2015, p.163).

Bajo la anterior definición y planteando la variedad de acciones que puede emprender el contador público en la organización privada, se establecieron ocho categorías en las cuales se desempeña este profesional:

- Cumplimiento legal: corresponde a la actualización y aplicación de la regulación que el profesional contable tiene a cargo en los diversos procesos donde interactúa al interior de la organización y frente a externos.

- TIC: se refiere al uso de las tecnologías de la información y comunicación para cumplir con los deberes contables en la organización.

- Informes contables: presentación de los diversos reportes que requieren los usuarios de la información derivados del sistema de información contable.

- Análisis de la información para crear valor agregado: actividades que trascienden la elaboración de reportes contables, pues permiten relacionar los diferentes resultados de la organización para tomar decisiones y reorientar la estrategia, según las necesidades del entorno (interno y externo).

- Teneduría de libros: práctica de registro de transacciones en los libros de contabilidad. 
- Contador como gerente de la información financiera: el profesional contable planea, hace, verifica y actúa como líder de los sistemas de información financiera dentro de la organización con la finalidad de que esta logre sus objetivos.

- Ética: corresponde al comportamiento del profesional en cumplimiento del código de buenas prácticas que debe aplicar para que sus actividades estén orientadas a presentar la realidad de lo que sucede con los recursos de la organización.

- Social de stakeholders: son las acciones en las cuales el contador público identifica y reporta información, comprendiendo los requerimientos de los distintos usuarios, con una mirada integral de responsabilidad social.

En la Figura 1, se resumen las categorías de análisis para las prácticas del profesional contable que ejerce en la empresa privada.

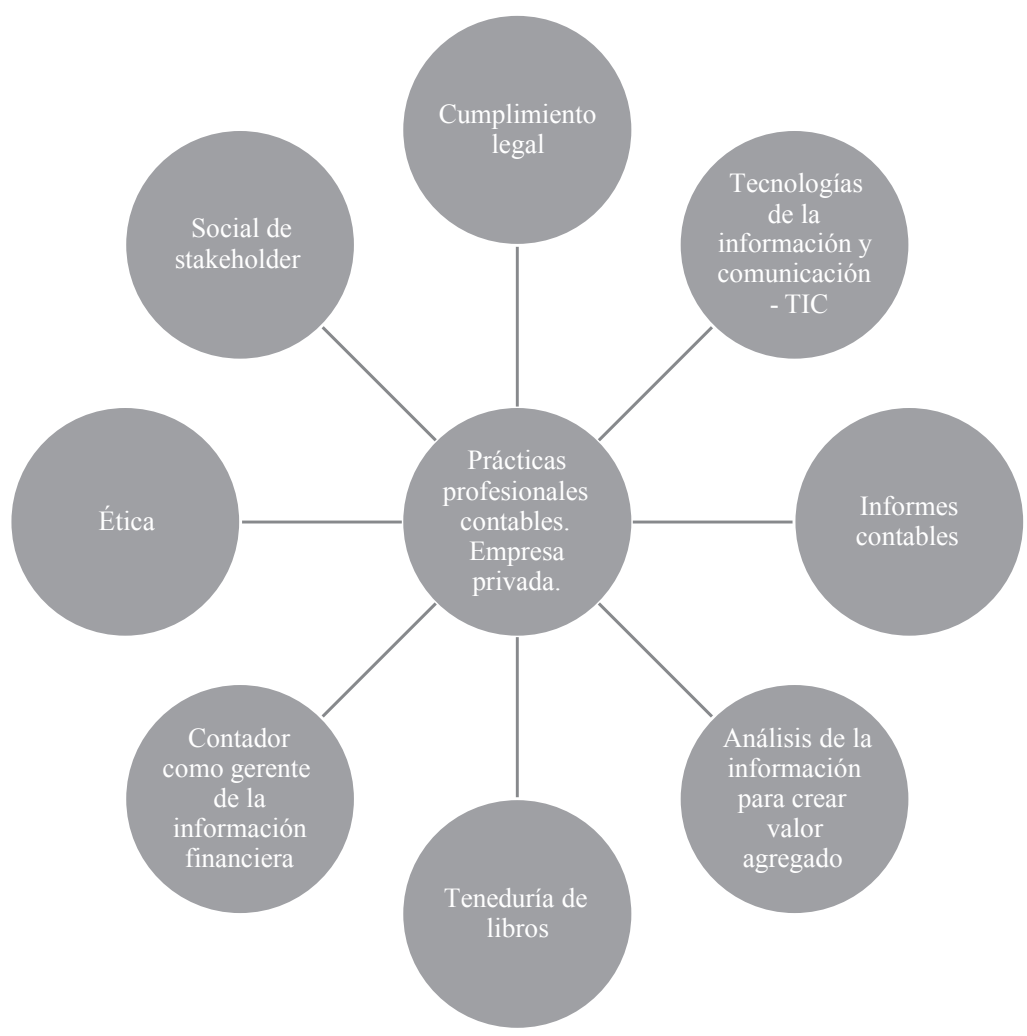

Figura 1. Prácticas profesionales contables en la empresa privada. Elaboración propia, a partir de Castaño et al., 2015. 


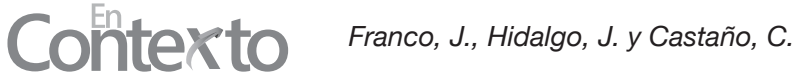

\section{Metodología}

Esta investigación es de tipo cualitativo, se fundamenta en análisis documentales (textos y normas) y entrevistas a profesionales de la contaduría que ejercen en entidades del grupo II para el sector privado, donde fue posible entrevistar a nueve contadores con más de cuatro años de experiencia. Se ha definido que sea para este grupo, dada la posibilidad de acceso a la información de primera mano de contadores laborando en este tipo de entidades y que, además, por el tiempo en ejercicio, tuviesen conocimiento de los acontecimientos en la profesión contable durante el período de evaluación. Se descartaron de la muestra los contadores del sector público, puesto que los tiempos de transición han sido diferentes; además, de que sus prácticas pueden tener algunas variaciones puntuales debido a las implicaciones de sus propios marcos regulativos especiales proferidos por la Contaduría General de la Nación.

En la estrategia de análisis documental, se partió de fuentes secundarias (libros, revistas, normas, prensa, trabajos de grado) lo que permitió conocer el estado actual de las prácticas contables bajo el nuevo marco normativo, la generación de los diferentes informes financieros y sus revelaciones, el alcance de estas frente a los stakeholders y su relación a través de la aplicación de la nueva normatividad contable. Otros aspectos estudiados fueron los conceptos stakehoders, enfoque social, bien común de la contabilidad y profesión contable. El trabajo se realizó para el período 2014-2018, pues en la investigación de Castaño et al. (2015) se estudió el período 1990-2013, y allí se estableció una línea base de análisis que permitió dar continuidad a la evaluación. En ese sentido, se recogieron las ocho perspectivas de análisis para revisar dicha evolución: perspectiva del cumplimiento legal, perspectiva de las TIC, perspectiva de informes contables, perspectiva del análisis de la información para crear valor agregado, perspectiva de la teneduría de libros, perspectiva del contador como gerente de la información financiera, perspectiva de la ética y perspectiva social de stakeholders.

Las entrevistas realizadas a los contadores iniciaron con una declaración de consentimiento informado, con lo cual se indicó que la información recolectada solo sería utilizada para fines académicos; posteriormente, se realizaron preguntas de contextualización y ambientación del tema; luego, preguntas sobre las diferentes perspectivas de análisis y, por último, unas preguntas de cierre e integración de conceptos (Anexo 1). 
Para seleccionar los contadores se inició con la validación previa, que cumplieran con las condiciones requeridas para el trabajo, que tuvieran más de cuatro años de experiencia y que estuvieran laborando en empresas que pertenecieran al grupo II de las Normas Internacionales de Información Financiera durante el período 2014-2018. En especial, se pensó en este tipo de empresas en donde el contador, por lo general, tiene a cargo la integridad del sistema contable, sea de forma individual o con un pequeño grupo de asistentes y auxiliares contables.

Frente al análisis de la información, el equipo investigador sistematizó y clasificó las respuestas, según las categorías propuestas. Estableció factores comunes que reiteraban los entrevistados. Asimismo, se pusieron de manifiesto algunos puntos relevantes que llamaron la atención a los investigadores y que se dejaron consignados a lo largo de los resultados.

\section{Resultados}

\section{Cumplimiento legal}

Franco (2011) expone que el ejercicio del profesional contable requiere, frente a la regulación, conocimiento de

[...] instituciones jurídicas, régimen de importaciones y exportaciones, instituciones políticas, régimen de contratación administrativa, legislación comercial, legislación laboral pública y privada, legislación tributaria comparada, nacional, departamental y municipal, legislación civil y legislación profesional nacional e internacional, pues todas estas normas controlan en mayor o menor grado el ejercicio de la profesión (p.259).

Conocimientos normativos que escapan a muchos contadores en su práctica profesional contable, debido, en algunos casos, a la poca fortaleza en la formación de este campo en las universidades; en otros, por el excesivo cambio regulativo en diferentes líneas, que hace que el contador se acomode solo a tratar de cumplir la norma sin entenderla (esto puede implicar sanciones para las organizaciones y para el contador público). Igualmente, el aceptar la aplicación de nuevos marcos normativos contables, sin analizar las situaciones nacionales e internacionales en el que interactúan las diferentes economías, puede generar un distanciamiento con la realidad económica de las empresas.

La mayoría de los entrevistados opina que en este caso la aplicación de las normas internacionales permitió un avance en los procedimientos de contabilización y 


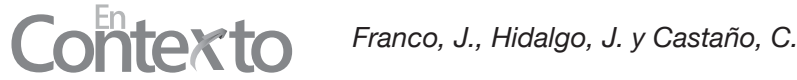

medición de los diferentes hechos económicos de las empresas. En la Tabla 1, se describe la evolución regulativa de la contabilidad en Colombia en el período 2014-2018 para empresas del grupo II.

Tabla 1

Evolución regulativa de la contabilidad en Colombia en el periodo 2014-2018 para Empresas del grupo II

\begin{tabular}{|c|c|}
\hline Norma & Título \\
\hline Ley 1314 de 2009 & $\begin{array}{l}\text { Por la cual se regulan los principios y normas } \\
\text { de contabilidad e información financiera y de } \\
\text { aseguramiento de información aceptados en } \\
\text { Colombia, se señalan las autoridades competentes, } \\
\text { el procedimiento para su expedición y se } \\
\text { determinan las entidades responsables de vigilar } \\
\text { su cumplimiento. }\end{array}$ \\
\hline $\begin{array}{c}\text { Decreto } 3022 \text { de } \\
\text { diciembre } 27 \text { de } 2013\end{array}$ & $\begin{array}{l}\text { Por medio del cual se reglamenta la Ley } 1314 \text { de } \\
2009 \text { para el grupo } 2 \text {. }\end{array}$ \\
\hline Decreto 2267 de 2014 & $\begin{array}{l}\text { Por medio del cual se modifica parcialmente el } \\
\text { Decreto } 3022 \text { de } 2013 \text {. }\end{array}$ \\
\hline Decreto 2420 de 2015 & $\begin{array}{l}\text { Por medio del cual se expide el Decreto Único } \\
\text { Reglamentario de las Normas de Contabilidad, de } \\
\text { Información Financiera y de Aseguramiento de la } \\
\text { Información y se dictan otras disposiciones. }\end{array}$ \\
\hline Decreto 2496 de 2015 & $\begin{array}{l}\text { Por medio del cual se modifica el Decreto } 2420 \\
\text { de } 2015 \text { Único Reglamentario de las Normas de } \\
\text { Contabilidad, de Información Financiera y de } \\
\text { Aseguramiento de la Información y se dictan } \\
\text { otras disposiciones. }\end{array}$ \\
\hline Decreto 2101 de 2016 & $\begin{array}{l}\text { Por el cual se adiciona un título 5, denominado } \\
\text { Normas de Información Financiera para Entidades } \\
\text { que no Cumplen la Hipótesis de Negocio en } \\
\text { Marcha, a la Parte } 1 \text { del Libro } 1 \text { del Decreto } 2420 \\
\text { de } 2015 \text {, Único Reglamentario de las Normas } \\
\text { de Contabilidad, Información Financiera y de } \\
\text { Aseguramiento de la Información, y se dictan } \\
\text { otras disposiciones. }\end{array}$ \\
\hline Decreto 2131 de 2016 & $\begin{array}{l}\text { Por medio del cual se modifica parcialmente el } \\
\text { Decreto } 2420 \text { de } 2015 \text { modificado por el Decreto } \\
2496 \text { de } 2015 \text {, y se dictan otras disposiciones. }\end{array}$ \\
\hline
\end{tabular}




\begin{tabular}{cl}
\hline Norma & \multicolumn{1}{c}{ Título } \\
\hline & $\begin{array}{l}\text { Por medio del cual se modifican parcialmente los } \\
\text { marcos técnicos de las Normas de Información }\end{array}$ \\
& Financiera y de Aseguramiento de la Información \\
previstos en los artículos 1.1.1.2. y 1.2.1.1. Del \\
Lecreto 2170 de 2017 & los Decretos 2496 de 2015, 2131 y 2132 de 2016, \\
& respectivamente, y se dictan otras disposiciones. \\
\hline & Por medio del cual se compilan y actualizan los \\
& marcos técnicos de las Normas de Información \\
& Financiera NIIF para el Grupo 1 y de las Normas \\
& de Información Financiera, NIIF para las Pymes, \\
& Grupo 2, anexos al Decreto 2420 de 2015, \\
& modificado por los Decretos 2496 de 2015,2131 de \\
& 2016 y 2170 de 2017, respectivamente, y se dictan \\
& otras disposiciones. \\
\hline
\end{tabular}

Ministerio de Comercio, Industria y Turismo.

Hay que considerar en este punto, que si bien se atiende a la evolución de la regulación en la norma contable, también se han dado cambios en la regulación tributaria, laboral y comercial, que afectan el quehacer del profesional contable y que como se ha explicado, en estos casos, el profesional puede cometer errores en la aplicación, sea por la debilidad en su proceso formativo o porque el exceso de labores no le permite llevar a cabo un adecuado análisis de la labor que le corresponde.

\section{Las tecnologías de la información}

Según Castells (1998), lo que caracteriza a la revolución tecnológica actual es la información y la aplicación del carácter central del conocimiento a los aparatos que procesan dicha información, con lo que surge un círculo de retroalimentación entre la innovación y sus usos. Se presenta entonces como reto para el hombre de hoy (para las ciencias económicas, sociales, entre otras) el observar, analizar y teorizar sobre sus realidades como factores que ayudan en la transformación tecno-económica, y a construir un mundo diferente y mejor, en el cual, el hombre sea el centro de esa nueva visión, pero también el medio y el objetivo de una economía pensada en generar libertad y justicia.

Por otra parte, las tecnologías de la información, entendidas como el uso del hardware y software en la elaboración y preparación de los informes contables 


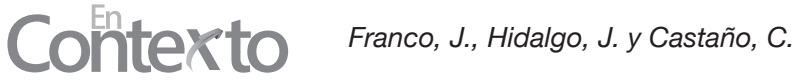

se desarrollaron con el objeto de propiciar al contador en su práctica contable una herramienta que realizara todos los cálculos y operaciones de una forma sistematizada con la mayor certidumbre posible, y que, a la vez, le permitiera dedicar más tiempo a entender y explicar los resultados financieros obtenidos. Sin embargo, dicha situación ha sido malograda, pues se evidencian dos escenarios negativos: el primero, con contadores de bajo conocimiento en el uso de las tecnologías y la segunda, contadores con amplio uso de tecnologías, pero recargados con mayor número de clientes lo que deriva en la continuidad de una práctica profesional muy mecánica al no contar con tiempo para profundizar en el análisis de datos.

En el caso de la implementación de los nuevos marcos técnicos normativos contables (derivados de la Ley 1314 de 2009) se requirió del desarrollo de nuevos programas o aplicaciones tecnológicas que permitieran la adecuación de los cambios en los procesos contables. Dichas aplicaciones requirieron del trabajo interdisciplinario de profesionales para hacer posible su transición a los nuevos estados financieros de apertura, cambios que aún no se logran visualizar concretamente en los planes de estudio de los programas de contaduría de las diferentes facultades en Colombia y que, adicionalmente, el contador que ya estaba en ejercicio difícilmente asimila. A pesar la situación, existen múltiples casos en los que el contador desempeñó un papel protagónico en las empresas para el cambio de sistema de contabilidad, al liderar grupos de trabajo en la elaboración de los estados financieros de apertura, recuperando su visibilidad profesional al interior de las organizaciones.

Así mismo, la transformación tecnológica que se ha dado mucho más fuerte en los últimos 20 años ha hecho evidente la necesidad de que las facultades de contaduría inserten en sus programas contables el uso de las TIC (Barreto et al., 2011). En este sentido, el profesor Castaño (2017) manifiesta que el uso de las tecnologías de la información y comunicación son cruciales en la redefinición del futuro de la educación contable, en especial, con referencia a temas como: big data, cloud computing, facturación electrónica, contabilidad en tiempo real (on-line). Sumado a esto, aparecen retos como la homogenización y comparabilidad de la información financiera, a través del Lenguaje Extensible de Reportes de Negocios (Extensible Business Reporting Language XBRL, en inglés), el cual permite que sean leídos y entendidos en cualquier lugar del mundo (Zamarra et al., 2017), elementos que simplemente no pueden dejarse de lado para el ejercicio profesional del contador. 
Con relación a esta dimensión de las prácticas contables, los entrevistados plantearon que efectivamente esta nueva regulación contable generó demanda de nuevos desarrollos tecnológicos y que el contador aún adolece de una buena formación en este campo, lo que afecta el ejercicio de sus prácticas profesionales. Este tipo de problema no obedece principalmente al conocimiento normativo, sino al conocimiento del desarrollo de la tecnología, donde los programas de formación del contador público colombiano poco consideran temas como el big data, machine learning, y en general, la programación de software.

\section{Informes contables}

El nuevo marco técnico normativo contable para empresas de grupos II indica que los estados financieros básicos son: el estado de situación financiera, el estado de resultados, el estado de flujos de efectivo y el estado de cambios en el patrimonio, y las notas a los estados financieros que hacen parte integral de estos. Este ajuste, trae consigo nuevos términos a utilizar e indicaciones a cumplir en la presentación y elaboración de cada estado financiero, conocimientos que al momento aún presentan dificultades en su entendimiento para el contador y el equipo de profesionales que hacen parte de su elaboración, dado que la implementación de las normas hizo posible que la dirección con todas las áreas o departamentos se involucraran en su elaboración. Dejando clara la responsabilidad de cada dependencia en el tratamiento de la información financiera, y su envío al área contable para su consolidación y presentación como único sistema de la empresa que contiene características propias que permiten identificar su posicionamiento y lectura ante los demás.

Los reportes contables han evolucionado hacia informes integrados (Integrated Reporting), promovidos por el Internacional Integrated Reporting Council (Consejo Internacional de Reporte Integrado) con un planteamiento de uso de información financiera y no financiera y el análisis de seis tipos de capital como son el financiero, industrial, intelectual, humano, social y relacional y natural, propendiendo por un desarrollo sostenible de las organizaciones.

Rivera-Arrubla et al. (2016) presentan una cronología que da cuenta de cómo estos reportes de contabilidad han evolucionado desde una mirada únicamente financiera, hacia la triple dimensión financiera, ecológica y social (Tabla 2), pues se espera que las organizaciones busquen el equilibrio de sus actuaciones frente a una sociedad que le exige cada vez mayor responsabilidad en su actuación. 
Tabla 2

Evolución del reporting sobre información financiera y no financiera

\begin{tabular}{|c|c|c|}
\hline Cronología & Tipos de informe & $\begin{array}{c}\text { Dimensiones } \\
\text { incluidas en el } \\
\text { contenido de los informes }\end{array}$ \\
\hline Antes de los años 70. & $\begin{array}{l}\text { Informes con contenido } \\
\text { financiero. }\end{array}$ & $\begin{array}{c}\text { Dimensión } \\
\text { exclusivamente financiera. }\end{array}$ \\
\hline \multirow[t]{2}{*}{$\begin{array}{l}\text { Década de los } \\
\text { años } 70 \text { y } 80 .\end{array}$} & $\begin{array}{l}\text { Informes aislados de } \\
\text { información financiera. }\end{array}$ & $\begin{array}{c}\text { Una dimensión aislada de } \\
\text { la información social y } \\
\text { ambiental. }\end{array}$ \\
\hline & $\begin{array}{c}\text { Informes aislados de } \\
\text { información social o } \\
\text { ambiental. }\end{array}$ & $\begin{array}{c}\text { Una dimensión de } \\
\text { sostenibilidad (social o } \\
\text { ambiental). }\end{array}$ \\
\hline Década de los 90. & $\begin{array}{l}\text { Informes aislados de } \\
\text { información financiera. } \\
\text { Informes especializados } \\
\text { en sostenibilidad que } \\
\text { conjugan información } \\
\text { social y ambiental. }\end{array}$ & $\begin{array}{l}\text { Dimensión financiera. } \\
\text { Dos dimensiones de } \\
\text { sostenibilidad que ganan } \\
\text { vigor: ecología y social. }\end{array}$ \\
\hline Década del 2000. & $\begin{array}{l}\text { Algunas iniciativas de } \\
\text { combinar información } \\
\text { de sostenibilidad e } \\
\text { información financiera } \\
\text { bajo el informe anual. }\end{array}$ & $\begin{array}{l}\text { Recuperación de la } \\
\text { importancia de la } \\
\text { dimensión financiera } \\
\text { de los informes y auge } \\
\text { de la información con } \\
\text { contenido no financiero. }\end{array}$ \\
\hline Tendencia actual. & $\begin{array}{l}\text { Un único Informe } \\
\text { Integrado } \\
\text { (visión holística de la } \\
\text { organización). }\end{array}$ & $\begin{array}{c}\text { Tres dimensiones } \\
\text { de sostenibilidad } \\
\text { (financiera, ecológica y } \\
\text { social en equilibrio). }\end{array}$ \\
\hline
\end{tabular}

Rivera-Arrubla et al., 2016, p.149.

Los entrevistados manifiestan que los informes contables han cambiado en su contenido y presentación al incluirse más detalle para su elaboración con el fin de contribuir a su entendimiento y a que sirva para la toma de decisiones, un aspecto de transformación de sus prácticas que conlleva un trabajo mucho más amplio que el que anteriormente se realizaba. 


\section{Análisis de información para crear valor agregado}

El contador público con su equipo de trabajo dedicaba sus esfuerzos a cumplir con la recopilación de datos de las diferentes áreas que conforman la empresa con el solo objetivo de cumplir con los diferentes informes en los tiempos establecidos por la dirección y las diferentes entidades de regulación y control. Es así como Becerra (2016) plantea que el rol del contador público ha cambiado y se espera que tenga una participación en la formulación, implementación y seguimiento de la estrategia organizacional. Además del apoyo en la gestión de riesgos y una mirada que combine factores económicos, sociales y ambientales, lo cual redunda, en el apoyo a la generación del valor organizacional.

Este aspecto de la generación de valor se convierte crucial para la profesión contable, toda vez que las organizaciones requieren un profesional activo en temas como: costos, fijación de precios, presupuestos, planeación financiera y fiscal, control interno, entre otros; ámbitos que repercuten en la posibilidad de mantener a las organizaciones en marcha, pero que, a su vez, tienen incidencia en cómo se construye la sociedad. Frente a este último punto, cabe la pena anotar la relevancia de crear valor social, con una mirada de sustentabilidad futura desde el accionar organizacional en interacción con el medio ambiente y la humanidad.

Los entrevistados coinciden en que, existen avances en esta materia, pero hace falta mayor capacitación en los contadores públicos y sensibilización para lograr cabalmente la generación de valor y, por otro lado, queda la sensación de que persiste en la gerencia de las MIPYMES una mirada de la contabilidad para el cumplimiento legal, razón por la cual, los gerentes de estas entidades no se preocupan por capacitarse para comprender de mejor manera la información que el contador les entrega.

\section{Teneduría de libros}

La teneduría de libros se refiere a la tarea rutinaria del reconocimiento, clasificación y registro de operaciones, principalmente de orden financiero, en las organizaciones. Este concepto de teneduría es común evidenciarlo en textos clásicos de historia y enseñanza de la contabilidad comercial para las universidades, es así como la Contaduría General de la Nación (2010), en su diccionario de términos de contabilidad pública, lo define como "[...] actividad que tiene por objeto registrar, clasificar y resumir las operaciones transaccionales del ente contable, manteniendo con el mayor orden y claridad tales registros" (p.110). 


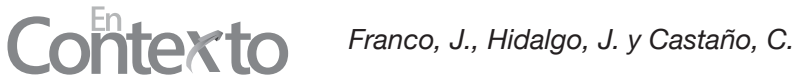

La mayoría de los contadores entrevistados tienen claro que la teneduría de libros o registros contables son meramente una etapa del proceso, pero que no es lo más importante, dado el apoyo de los sistemas computarizados. También consideran que el término teneduría de libros está obsoleto, pues lo que se busca es darle valor agregado a la información y que el proceso contable implica más que una simple teneduría de libros. Sin embargo, algunos consideraron que se continúa con la teneduría de libros sobre todo en las empresas más pequeñas.

\section{El contador como gerente de información financiera}

De acuerdo con la percepción de las personas entrevistadas, el contador ya no solo se dedica a llevar registros contables, sino que adquiere un perfil más financiero, velando porque la información sea precisa y sirva para la toma de decisiones. La capacitación es fundamental para realizar las nuevas prácticas contables basadas en estándares internacionales, además de considerar que la responsabilidad de la información financiera no es únicamente del contador, la función del contador es operar la información que recibe de cada uno de los responsables de los procesos en las organizaciones. Por eso, es importante sensibilizar al personal e integrarlo a las prácticas corporativas.

\section{La ética}

Según Martínez (2006), la ética profesional es una fuerza que orienta los comportamientos de las personas frente a lo que mueve al profesional y lo que legítimamente demanda la comunidad a los profesionales. También manifiesta que en cada profesión se elabora una ética específica que es revisada y puesta al día periódicamente, pero las distintas éticas deben respetar y apoyar el marco ético como verdadero soporte moral de la convivencia en las sociedades.

Vargas y Católico (2007) manifiestan que el contador público debe liderar el comportamiento ético, toda vez que su función social pueda beneficiar o no a toda una comunidad. En este sentido, este profesional debe actuar acorde a las normas establecidas en el código de ética profesional, pero, ante todo, con gran responsabilidad e integridad para mantener alejadas de las organizaciones los actos que atentan contra el interés común.

Por su parte, Agudelo y Viloria (2017) expresan que la práctica contable debe estar permeada por virtudes de los profesionales que ejercen la contaduría y proponen que sean la honestidad, la justicia, el valor y prudencia los ejes de esas acciones que el profesional contable va a desarrollar, pues el propósito de 
proteger el interés público no se logra únicamente con el establecimiento de un código de ética y verificación a modo de lista de chequeo, sino que se requiere educar y motivar al profesional para que actúe bajo las virtudes propuestas.

Es importante resaltar entonces, otros aspectos que complementan una actitud ética del contador: la educación contable, la competencia profesional, el desarrollo profesional continuo, el conocimiento en control, auditoría, riesgos y el juicio profesional (independencia y criterio). Aspectos que son de gran interés por los diferentes organismos contables internacionales, quienes participan en la elaboración de los diferentes códigos de ética, en especial, el producido por IFAC (Federación Internacional de Contadores, por sus siglas en inglés) que ya se convirtió en norma para los contadores colombianos desde la entrada en vigencia del decreto reglamentario 302 de 2015; también trajo consigo el estándar de control de calidad (López y Gómez, 2018) que quedó compilado y actualizado a través del decreto único reglamentario 2420 de 2015 y posteriores.

Se infiere de las entrevistas que las prácticas contables orientadas en la ética no han sufrido cambios, que dichas prácticas son inherentes a las personas, pero piensan que los profesionales de la contabilidad se han hecho más conscientes de la responsabilidad que conlleva su labor. Allí, se vincula el comportamiento a lo cultural e indican que, si en la cultura empresarial falta ética, esto se hará recíproco en el ejercicio de la profesión. Así mismo, plantean que la regulación se ha tornado más exigente en los últimos cuatro años; sin embargo, algunos aseguran que hace falta reforzar el tema de gobierno corporativo, ${ }^{1}$ como una forma de mostrar las buenas prácticas empresariales entre la administración, accionistas, empleados, Estado y comunidad.

\section{Perspectiva social de stakeholders}

De acuerdo con la mayoría de las personas que formaron parte del estudio, el profesional contable ya no es un sujeto encerrado en una oficina. Por los cambios que se vienen dando, es necesario el contacto constante con los diferentes interesados, tanto internos como externos, al tener nuevos estándares y ampliar los usuarios de la información, con la globalización y los nuevos negocios,

\footnotetext{
Gobierno corporativo es "[...] la defensa de los intereses de los accionistas (Tirole, 2001); en este sentido, el gobierno corporativo se estudia desde la separación entre la propiedad y el control, en la que los administradores actúan como agentes de los inversionistas (accionistas y tenedores de bonos) para decidir las inversiones a realizar y la forma en que se van a financiar estas inversiones, mientras que la junta directiva se encarga de gobernar y controlar la gestión" (Lagos et al., 2018, p.88).
} 


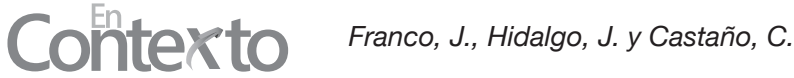

la generación de información cumple un papel mucho más importante para el profesional contable por la contribución de esta en la toma de decisiones de los stakeholders.

Existen elementos que conducen a determinar la importancia de la labor del contador a través del e jercicio de las prácticas contables, dada la responsabilidad que se le atribuye para que sirva de garante ante la sociedad, los empresarios, los trabajadores y el Estado.

Entre estos elementos se pueden enunciar: el pago debido a la seguridad social (salud, pensiones y ARL), la certificación del uso del software con licencias en las empresas, el registro real de los hechos económicos que realiza la empresa (compras, ventas, importaciones y exportaciones entre otros), el pago adecuado y oportuno de los diferentes impuestos al Estado, el pago justo a los empleados con sus prestaciones sociales, el cumplimiento de las normas por parte de la empresa, la elaboración con transparencias de los diferentes informes financieros de la empresa, que den fe de su estado real, la elaboración de productos o prestación de servicios con el cumplimiento de las características contenidas en ellos, el uso adecuado de las diferentes herramientas y maquinarias elaboradas con tecnologías limpias que contribuyan a minimizar el impacto con el medio ambiente, el cumplimiento oportuno de las obligaciones contraídas con todos los vinculados económicos, el control del vertimiento de contaminantes a los ríos y afluentes, entre otros, aspectos que, contribuyen a un desarrollo sostenible de las empresas, de la comunidad y, por ende, de los países y que demuestran la responsabilidad social que tiene el contador en su ejercicio profesional.

El no hacerlo, es conducir a un país a una quiebra o estancamiento, como ha sucedido en tiempos pasados en el exterior y en Colombia cuando los medios informativos encabezaban las noticias con preguntas como: ¿dónde están los revisores fiscales frente a la liquidación de tantas empresas?

\section{Análisis integrado de la evolución de las prácticas contables profesionales}

En la Tabla 3, se describe la evolución de las prácticas contables del contador en el relacionamiento con los stakeholders para el período de estudio. 
Evolución de las prácticas profesionales contables ...

Tabla 3

Evolución de las prácticas contables del contador en el relacionamiento con los stakeholders período 2014-2018

\begin{tabular}{ll}
\hline Perspectiva & \multicolumn{1}{c}{ Análisis } \\
\hline & La mayoría de los contadores entrevistados plantea que las \\
prácticas sí han evolucionado en el periodo 2014 a 2018, como & consecuencia de la emisión de la ley de convergencia, los \\
& marcos técnicos normativos de la contabilidad financiera, las \\
& normas de aseguramiento de información financiera, y todas \\
& las normas relacionadas, además de la llegada con fuerza de la \\
& necesidad de manuales de políticas contables mucho mejores \\
Cumplimiento legal & que los que se venían preparando y los ajustes en cultura \\
& organizacional. Hay que considerar, además, que la regulación \\
& en aspectos como los tributarios, comerciales y laborales, entre \\
& otros, que se relacionan con la labor del contador, presentan \\
& constantes cambios y esto requiere actualización permanente \\
& del profesional contable. Frente a este asunto, solo uno de los \\
& entrevistados consideró que las prácticas no han evolucionado, \\
& y otro consideró que las prácticas no evolucionaron de manera \\
& consciente, sino forzada. \\
\hline & Los entrevistados consideran que las tecnologías van \\
& cambiando a medida que la reglamentación, las normas \\
& contables y tributarias cambian, pues estas exigen nuevos \\
& requerimientos, además cada día se deben manejar volúmenes \\
& de información más grandes que exigen nuevos desarrollos \\
& tecnológicos que faciliten su procesamiento y así poder \\
& cumplir con las exigencias de informes de los entes de control \\
& y demás usuarios, sumado a esto los desarrollos también \\
& deben buscar integralidad y control de la información. Sin \\
embargo, preocupa la falta de formación y relacionamiento \\
del contador con estos temas.
\end{tabular}

La mayoría de los entrevistados considera que la preparación y elaboración de los informes contables han variado por la adopción de la nueva regulación contable en cuanto al reconocimiento de los elementos de los estados financieros y la preparación de los reportes. Además los requisitos contenidos en las normas buscan que "la información sea más clara, precisa y acorde con la realidad de las empresas"

Informes contables (tomado de una de las entrevistas); también considera que con la implementación de los estándares internacionales nacen requisitos que buscan hacer de la contabilidad una herramienta más financiera que fiscal, algunos entrevistados consideran que en las PYMES y en las microempresas no se han presentado mucha diferencia en la elaboración de la información, más allá de que se incorpora nueva terminología en los estados financieros y algunos elementos financieros. 
Perspectiva

Análisis de la información para crear valor agregado

La mayoría de los entrevistados consideran que la información contable sí crea valor siempre y cuando los contadores sean éticos y estén capacitados en las nuevas normas, plantean también que la información es más precisa y ajustada a la realidad económica de la empresa, además se ha agregado más conciencia de la información y su uso. Algunos entrevistados manifiestan la existencia de avances, pero para las PYMES y microempresas todavía falta mucho por avanzar y generar valor, ya que en la mayoría de los casos la alta gerencia no se preocupa en capacitarse y ven la contabilidad como una norma que se tiene que cumplir simplemente.

La mayoría de entrevistados consideró que el término teneduría de libros es un término obsoleto, pues lo que se busca es darle

Teneduría de libros valor agregado a la información, manifestaron que el proceso contable implica más que una simple teneduría de libros y algunos consideraron que se continua con la teneduría de libros sobre todo en las empresas de menor tamaño.

Si el contador ya no solo se dedica a llevar registros contables, sino que adquiere un perfil más financiero, velando porque la información sea precisa y sirva para la toma de decisiones, la capacitación es fundamental para realizar las nuevas Gerente de la información prácticas contables basadas en estándares internacionales de información financiera, además de considerar que "la financiera responsabilidad de la información financiera no es únicamente del contador. La función del contador es operar la información que recibe de cada uno de los responsables de los procesos en las organizaciones. Por eso es importante sensibilizar al personal e integrar las prácticas corporativas" (tomado de una de las entrevistas).

Los entrevistados piensan que las prácticas contables orientadas en la ética no han sufrido cambios, que dichas prácticas son inherentes a las personas, pero piensan que los Ética profesionales de la contabilidad se han hecho más conscientes de la responsabilidad que conlleva su labor; sin embargo, un entrevistado piensa que "la ética como práctica es algo cultural como sociedad. Si en la cultura empresarial falta ética en el ejercicio de la profesión también habrá falta de ética".

La mayoría de entrevistados piensan que el profesional contable ya no es un sujeto encerrado en una oficina, por

Relacionamiento con los stakeholders los cambios que se vienen dando es necesario el contacto constante con los diferentes interesados, al tener nuevos estándares y ampliar los usuarios de la información, con la globalización y los nuevos negocios, la información cumple un papel mucho más importante en la toma de decisiones.

Elaboración propia con los resultados obtenidos a través de las entrevistas. 
Frente a los resultados obtenidos por Castaño et al. (2015), se evidencian notables cambios en aspectos como:

- Cumplimiento legal: cambio total de la regulación contable financiera y una constante actualización de los marcos técnicos contables frente a los cambios que va estableciendo el emisor internacional.

- TIC: mayor incorporación de la tecnología para dar respuesta a la nueva regulación y necesidades de información de los distintos usuarios de la información.

- Informes contables: cambio en algunos elementos de conceptualización, pero para las MIPYMES no hubo grandes ajustes.

- Análisis de la información para crear valor agregado: es necesario crear un mejor ambiente para que estas prácticas, que puede desarrollar el profesional contable, lleguen a las empresas más pequeñas, siempre y cuando se genere un cambio importante en la cultura empresarial.

- Teneduría de libros: se considera un concepto obsoleto, aunque en las empresas de menor tamaño aún el profesional contable se centra en este tipo de práctica.

- Gerente de la información financiera: como la información financiera ya no se considera solo responsabilidad del contador, se ha avanzado un poco para que el profesional contable pueda liderar más que operar.

- Ética: se considera inherente a la profesión y cultura. Sin embargo, se denota un endurecimiento de la regulación y las responsabilidades del profesional contable. Algo que ya se venía notando en el anterior trabajo y que se profundiza en este estudio.

- Relacionamiento con los stakeholders: se trasciende de la mirada de responsabilidad social solo de la empresa y se considera que en sí mismo el contador público es socialmente responsable y requiere pensar en los interesados de la información. Se incrementa el uso de informes sociales y ambientales.

Como se puede evidenciar, en el proceso de transición hacia el nuevo marco normativo contable durante el período 2014-2018, en términos generales el profesional contable reavivó sus conocimientos profesionales, incrementó su acercamiento con las empresas donde ejerce su función tanto al interior como al exterior de las mismas, dado que este proceso obligó a que los contadores mejoraran en sus prácticas profesionales contables los procesos para la elaboración y generación de informes de tal forma que satisficieran a todas las personas interesadas. 


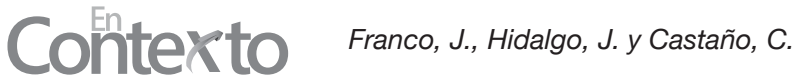

\section{Conclusiones}

La contaduría pública ha tenido una evidente evolución, en los periodos propuestos para este artículo, debido a las demandas presentadas en las diferentes empresas y los cambios tecnológicos que favorecen su acción. Igualmente, al crecimiento económico y la internacionalización de la economía que ha obligado a que adecúe sus métodos a las necesidades de las compañías, el Estado y los inversores, y en general a los stakeholders. Es por esto que cuando todos los actores involucrados en la profesión contable trabajan mancomunadamente, se puede mantener prácticas contables actualizadas que permitan atender las exigencias de los diferentes grupos de interés.

En el ejercicio de las prácticas profesionales se requiere identificar grupos de interés más allá de los socios de las empresas, el Estado y las entidades financieras, con el fin de propiciar los informes que estos requieren para el cometido de sus intereses. Bajo esta premisa el contador público contribuye al desarrollo armónico de la sociedad.

El cambio desde una praxis técnica hacia la gestión de la información integral en las organizaciones plantea un contador que no solo requiere educación integral, sino un ejercicio profesional enmarcado en las mejores prácticas aplicables, según los tipos de entidades en donde se desempeñe. Además, la profesión necesita de una mejor reflexión sobre la ética profesional, la formación integral de los contadores públicos y la profundización sobre conceptos de cómo se puede generar valor desde los sistemas contables para los distintos stakeholders. Este trabajo de investigación propone que se continúe con la evaluación de las prácticas contables. Será necesario mirar cómo pueden variar para otros contextos el desempeño profesional del contador público tanto en el sector público, las entidades sin ánimo de lucro, como también el profesional que se desempeña en contabilidad de costos y gestión, el asesor tributario, entre otros roles. 


\section{Referencias}

Agudelo, M., y Viloria, N. (2017). Las virtudes de la práctica contable. Un análisis desde MacIntyre. Revista Argentina de Investigación en Negocios, 3(1), 59-72. http://ppct.caicyt.gov.ar/index.php/rain/article/ view/V3N1-2a06/10175

Ámez, F. (1999). Diccionario de contabilidad y finanzas. Cultural de Ediciones.

Araujo, J. (1995). La contabilidad social. Implicar.

Barreto, O., Cárdenas, S., y Mondragón, S. (2011). Las tecnologías de información y comunicación en la formación de contadores públicos: análisis de uso y aplicaciones en cinco universidades colombianas. Cuadernos de Contabilidad, 12(30), 243-272.

Becerra, G. (2016). El nuevo rol del contador público en las organizaciones [Trabajo de pregrado]. Universidad Militar Nueva Granada. https:// repository.unimilitar.edu.co/bitstream/handle/10654/14631/ BecerraCamargoGabrielAndres.pdf? sequence $=1$ \&isAllowed $=y$

Castaño, C. (2017). Algunos aspectos sobre el futuro de la educación contable. Contaduría Universidad de Antioquia, 70, 11-12.

Castaño, C., Carmona, M., Mesa, M., y Muñetón, N. (2015). Prácticas profesionales contables en la empresa privada colombiana 1990-2013. Revista En-Contexto, 4(4), 15 9-184.

Castells, M. (1998). La era de la información: economía, sociedad y cultura. Entender nuestro mundo. Alianza Editorial.

Congreso de la Republica. (13 de diciembre de 1990). Ley 43 de 1990. Número 39602.

Congreso de la República. (13 de julio de 2009). Ley 1314. Número 51478.

Contaduría General de la Nación. (2010). Diccionario de términos de contabilidad pública. CGN.

Decreto 2131. (22 de diciembre de 2016). Decerto Ordinario. Número 50096. 


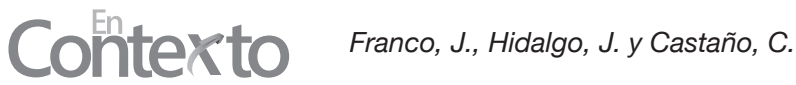

Decreto 2170. (22 de diciembre de 2017). Decreto Reglamentario. Número 50455.

Decreto 2420. (14 de diciembre de 2015). Normas de contabilidad, de información financiera y de aseguramiento de información.

Decreto 2267. (11 de noviembre de 2014). Decreto Reglamentario. Número 49332.

Decreto 2496. (23 de diciembre de 2015). Decreto Reglamentario. Número 49735.

Decreto 3022. (27 de diciembre de 2013). Código de Comercio.

Franco, R. (2011). Reflexiones contables. Universidad Libre de Colombia.

Freeman, R. (1984). Strategic management: A stakeholder approach. Pitman.

Gertz Manero, F. (1976). Origen y evolución de la contabilidad. Editorial Trillas.

Hernández, E. (2002). La historia de la contabilidad. Revista libros, 67-68. https:// www.aeca.es/old/comisiones/historia/lahistoriadelacontabilidad.htm

Lagos, D., Betancourt, J., y Gómez, G. (2018). Relación entre gobierno corporativo, control familiar y desempeño en empresas colombianas. INNOVAR. Revista de Ciencias Administrativas y Sociales, 28(69), 85-98.

Larrinaga, C. (2017). En torno a la evolución de la investigación en Contabilidad Social y Medioambiental. Teuken Bidikay, 8(11), 21-38.

López, O., y Gómez, L. (2018). Diagnóstico de la implementación en Colombia, de las Normas Internacionales de Control de Calidad y del Código de Ética, en el trabajo de los revisores fiscales en las organizaciones del grupo 2. Revista En-Contexto, 6(9), 181-201.

MacIntyre, A. (2004). Tras la virtud. A\&M Graphics.

Martínez, E. (2006). Ética de la profesión: proyecto personal y compromiso de ciudadanía. Revista Veritas, 14, 121-139. 
Ministerio de Comercio. (22 de diciembre de 2016). Decreto 2101.

Ministerio de Comercio. (28 de diciembre de 2018). Decreto 2483. Información Finaciera NIIF.

Reckwitz, A. (2002). Toward a theory of social practices: a development in culturalist theorizing. European Journal of Social Theory, 5(2), 243-263. DOI: $10.1177 / 13684310222225432$.

Richardson, A. (2018). The accountancy profesión. R. Roslender (Ed.), The Roudledge Companion to Critical Accounting (Chapter 8). Roudledge Taylor \& Francis.

Rivera, N. (2013). La contabilidad al servicio de la sociedad, una memoria deformada. Contaduría Universidad de Antioquia, 63, 97-126.

Rivera-Arrubla, Y., Zorio-Grima, A., y García-Benau, M. (2016). El concepto de informe integrado como innovación en reporting corporativo. Journal of Innovation \& Knowledge, 1, 144-155.

Sy, A. (2014). Sepultar a Pacioli en África. La cosificación de la Contaduría por un tenedor de libros. Teuken-Bidikay, 5, 25-54.

Vargas, C., y Católico, D. (2007). La responsabilidad ética del contador. Activos, 7(12), 1-13.

Zamarra, J., Atehortúa, T., y Sierra, V. (2017). Homogenización y comparabilidad de la información financiera a través del XBRL. Contaduría Universidad de Antioquia, 71, 213-231.

\section{Para citar este artículo:}

Franco, J., Hidalgo, J. y Castaño, C. (2020). Evolución de las prácticas profesionales contables en el relacionamiento de los stakeholders, 2014-2018. En-Contexto, 8(13), 197-223. 


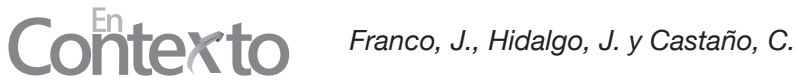

\section{Anexo 1}

Guía de entrevista

La información recolectada tiene como fin presentar el estado en el que se encuentran los informes elaborados por los contadores públicos a través de la práctica contable de 2014-2018 y su relación o atención que tienen estos informes con los stakeholders. Igualmente se aclara que la información tomada a través de este cuestionario solo es para fines académicos y en ningún caso los resultados determinarán o revelarán nombres específicos.

\section{Contextualización}

1. ¿Cuál es su cargo dentro de la empresa?

2. ¿Cuáles son los roles contables que ejerce como contador independiente?

3. ¿Cuántos años de experiencia lleva como contador? (Para efectos del proyecto se requiere que lleve mínimo cuatro años para que hable de los cambios)

4. ¿Para usted qué es una práctica contable?

5. ¿Qué entiende por stakeholders?

6. ¿Qué relación existe entre las prácticas contables y la información que se le debe brindar a esos stakeholders?

7. Para usted, ¿cuáles son las principales responsabilidades del contador público con la sociedad?

\section{Preguntas por categorías}

Uno de los intereses de este estudio es notar si dentro de las prácticas contables ha existido alguna evolución en el período que comprende entre los años 2014 a 2018. Para ello, haremos algunas preguntas desde diferentes ámbitos y queremos que nos describa si desde su experiencia estas prácticas han tenido alguna modificación sea esta positiva o negativa o si considera que sigue siendo igual.

1. Desde el cumplimiento de la regulación, ¿considera usted que han evolucionado las prácticas del contador en el período 2014 a 2018 ?

2. ¿Considera que ha cambiado algo esta actividad en el período 2014 a 2018 para sus prácticas contables?

3. ¿Ha variado la preparación o elaboración de informes contables en el período 2014-2018? ¿En qué sentido?

4. ¿Considera que esto ha cambiado en el período 2014-2018? 
5. ¿Considera usted que todavía podemos hablar de teneduría de libros o esto se ha modificado en el período 2014-2018?

6. La práctica contable del contador como gerente de información financiera en las organizaciones, ¿ha tenido algún cambio en el período 2014-2018?

7. ¿Ha sufrido alguna modificación de las prácticas contables con respecto a la ética del contador público en el período 2014-2018? ¿Se aplica la Ley 43 de 1990, tal como está estipulado?

8. Desde una aproximación a los diversos agentes (stakeholders) que intervienen con las organizaciones, ¿considera usted que se ha modificado la forma en que el contador puede relacionarse con estos, en el período 2014-2018?

\section{A. Cierre de las preguntas}

1. ¿Piensa que en general los cambios o situaciones anteriormente mencionadas han hecho que el contador público pueda mejorar su relacionamiento con los diferentes stakeholders o si por el contrario se ha deteriorado este relacionamiento en el período 2014-2018?

2. En su opinión, ¿qué se podría hacer para que los contadores públicos sean socialmente más responsables?

3. ¿Desea complementar algo frente a la temática que hablamos el día de hoy? 